\title{
Coherent multiple scattering in disordered media
}

\author{
E. Akkermans ${ }^{1}$ and G. Montambaux ${ }^{2}$ \\ ${ }^{1}$ Department of Physics, Technion, 32000 Haifa, Israel \\ ${ }^{2}$ Laboratoire de Physique des Solides \\ Université Paris-Sud \\ Bat.510, F-91405 Orsay Cedex, France
}

October 26, 2018

\begin{abstract}
These notes contain a rapid overview of the methods and results obtained in the field of propagation of waves in disordered media. The case of Schrödinger and Helmholtz equations are considered that describe respectively electrons in metals and scalar electromagnetic waves. The assumptions on the nature of disorder are discussed and perturbation methods in the weak disorder limit are presented. A central quantity, namely the probability of quantum diffusion is defined and calculated in the same limit. It is then shown that several relevant physical quantities are related to the return probability. Examples are provided to substantiate this, which include the average electrical conductivity, its fluctuations, the average albedo and spectral correlations.
\end{abstract}

\section{Introduction}

The study of wave propagation in random media gave rise to a huge amount of work especially during the last twenty years. Today, this field is split in two main subfields. One is concerned with the interplay between coherence and disorder in metallic systems and the second deals with the same problematics but for electromagnetic waves (this includes as well sound or gravity waves). Each one of the subfields has its own specificities and advantages, such as the effect of magnetic fields on transport and thermodynamics, interactions for metals and angular structure (spectroscopy) for waves. Unfortunately, the split between these two subfields has been growing so that their interplay is now certainly too weak in spite of the existence of a number of excellent reviews. These notes represent a very preliminary step towards a unified presentation of this field [1]. We have tried to present a general formalism that can apply to both situations (electrons and waves). It is centered around the existence of a basic quantity the probability of quantum diffusion which allows to describe either weak localization effects of the electrical conductivity of metals, spectral quantities of isolated electronic systems or the coherent and incoherent albedo, dynamical effects in multiple scattering of light by suspensions etc.

The next three sections contain basic definitions and generalities on the kind of waves we consider and the model of disorder. The section 5 deals with the definition and the calculation of the probability of quantum diffusion in multiple scattering using various approximations. Then, the subsequent sections apply these results to a selection of examples taken either from electronic systems (conductivity and spectral correlations) or multiple scattering of electromagnetic waves (average albedo in optical systems).

\section{Models for the disordered potential}

We shall consider mainly two problems where waves and disorder are involved. The first one corresponds to the study of spinless electrons in disordered metals or semiconductors. For a 
degenerate gas of free electrons of mass $m$ and charge $-e$, the Schrödinger equation is governed by the Hamiltonian

$$
\mathcal{H} \psi(\mathbf{r})=-\frac{\hbar^{2}}{2 m}\left(\nabla+\frac{i e}{\hbar} \mathbf{A}\right)^{2} \psi(\mathbf{r})+V(\mathbf{r}) \psi(\mathbf{r}) .
$$

where $\mathbf{B}=\nabla \times \mathbf{A}$ is the magnetic field. Effects associated to the band structure or to interactions between the electrons in the framework of Fermi liquid theory are accounted for by the replacement of the mass $m$ by an effective mass. The potential $V(\mathbf{r})$ decribes both the scattering by inhomogeneities and the confinement potential.

The second problem we shall consider is the propagation of electromagnetic waves of frequency $\omega$ in the scalar approximation. The behaviour of the electric field $\psi(\mathbf{r})$ is obtained from the Helmholtz equation

$$
-\Delta \psi(\mathbf{r})-k_{0}^{2} \mu(\mathbf{r}) \psi(\mathbf{r})=k_{0}^{2} \psi(\mathbf{r})
$$

where the function $\mu(\mathbf{r})=\delta \epsilon / \bar{\epsilon}$ is the relative fluctuation of the dielectric constant, $k_{0}=\bar{n} \frac{\omega}{c}$ and $\bar{n}$ is the average optical index. Under this form, the Helmholtz equation has a structure similar to the Schrödinger equation and the waves are scattered by the fluctuations of the dielectric function. It is nevertheless interesting to notice that in the latter case, the strength $\mu$ of the potential is multiplied by the frequency $\omega^{2}$ so that in contrast with electronic systems, a decrease of the frequency $\omega$ leads to a weaker effect of the disorder.

To account for the effects of the disorder in either case, we shall consider a random continuous function $V(\mathbf{r})$, of zero spatial average, $\langle V(\mathbf{r})\rangle=0$, where $\langle\cdots\rangle$ represents the spatial disorder average. The potential is characterized by its correlations especially by the two-point correlation function

$$
\left\langle V(\mathbf{r}) V\left(\mathbf{r}^{\prime}\right)\right\rangle=B\left(\mathbf{r}-\mathbf{r}^{\prime}\right)
$$

For a potential $V(\mathbf{r})$ which is localized enough, there exits a length $r_{c}$ which describes the fall off of $B\left(\mathbf{r}-\mathbf{r}^{\prime}\right)$. In the limit where the wavelength $\lambda \gg r_{c}$, the scattering events are statistically independent and we can consider the limiting case

$$
\left\langle V(\mathbf{r}) V\left(\mathbf{r}^{\prime}\right)\right\rangle=\gamma \delta\left(\mathbf{r}-\mathbf{r}^{\prime}\right)
$$

Such a potential is usually called a white noise. This is the case we shall consider throughout these notes. For the case of the Helmholtz equation, the potential is taken to be $V(\mathbf{r})=k_{0}^{2} \mu(\mathbf{r})$ so that $\gamma$, in that case, has the dimensions of the inverse of a length.

\section{Perturbation theory for multiple scattering}

\subsection{Single scattering - elastic scattering time}

For a very dilute system, we can first assume that a given incident wave of wavevector $\mathbf{k}$ is scattered only once into a state $\mathbf{k}^{\prime}$ before leaving the system. The lifetime $\tau_{k}$ of the state $\mathbf{k}$ is then given (using the notations of quantum mechanics) by the Fermi golden rule

$$
\frac{1}{\tau_{\mathbf{k}}}=\frac{2 \pi}{\hbar} \sum_{\mathbf{k}^{\prime}}\left|\left\langle\mathbf{k}|V| \mathbf{k}^{\prime}\right\rangle\right|^{2} \delta\left(\epsilon_{\mathbf{k}}-\epsilon_{\mathbf{k}^{\prime}}\right) .
$$

For the white noise case, we obtain

$$
\frac{1}{\tau_{e}}=\frac{2 \pi}{\hbar} \rho_{0} \gamma
$$

where $\rho_{0}$ is the density of states per unit volume. To this time, we can associate a length, the elastic mean free path $l_{e}$, defined as $l_{e}=v \tau_{e}$ by using the group velocity $v$. But the Fermi golden rule is valid for short times $t \ll \tau_{e}$ and in perturbation with the scattering potential $V$. In order to go beyond these limitations, we need to resort to a more powerful tool namely the formalism of the Green functions. 
The Green function associated to a wave equation can be defined as the response to a pulse i.e. to a $\delta$-function perturbation. For the case of the hamiltonian (1), the Green function $G^{R, A}\left(\mathbf{r}_{i}, \mathbf{r}, \epsilon\right)$ is solution of

$$
(\epsilon-\mathcal{H} \pm i 0) G^{R, A}\left(\mathbf{r}_{i}, \mathbf{r}, \epsilon\right)=\delta\left(\mathbf{r}-\mathbf{r}_{i}\right)
$$

We can define as well the free Green function $G_{0}\left(\mathbf{r}_{i}, \mathbf{r}, \epsilon\right)$ in the absence of scattering potential, which is the solution of

$$
\left(\epsilon+\frac{\hbar^{2}}{2 m} \Delta_{\mathbf{r}} \pm i 0\right) G_{0}^{R, A}\left(\mathbf{r}_{i}, \mathbf{r}, \epsilon\right)=\delta\left(\mathbf{r}-\mathbf{r}_{i}\right)
$$

so that the Green function $G^{R, A}\left(\mathbf{r}_{i}, \mathbf{r}, \epsilon\right)$ can be also expressed as a solution of the integral equation

$$
G\left(\mathbf{r}_{i}, \mathbf{r}, \epsilon\right)=G_{0}\left(\mathbf{r}_{i}, \mathbf{r}, \epsilon\right)+\int G\left(\mathbf{r}_{i}, \mathbf{r}^{\prime}, \epsilon\right) V\left(\mathbf{r}^{\prime}\right) G_{0}\left(\mathbf{r}^{\prime}, \mathbf{r}, \epsilon\right) d \mathbf{r}^{\prime}
$$

the solution of (8) is given (for $\mathrm{d}=3$ ) by

$$
G_{0}^{R, A}\left(\mathbf{r}_{i}, \mathbf{r}, \epsilon\right)=-\frac{m}{2 \pi \hbar^{2}} \frac{e^{ \pm i k R}}{R}
$$

with $\epsilon=\hbar^{2} k^{2} / 2 m$.

For the Helmholtz equation (2), we obtain a sequence of similar equations, but attention needs to be paid to the fact that the dispersion of the waves is now linear instead of quadratic for the Schrödinger case. The Green equation is

$$
\left(\Delta_{\mathbf{r}}+k_{0}^{2}(1+\mu(\mathbf{r}))\right) G\left(\mathbf{r}_{i}, \mathbf{r}, k_{0}\right)=\delta\left(\mathbf{r}-\mathbf{r}^{\prime}\right)
$$

while the solution of the free Green equation obtained for $\mu(\mathbf{r})=0$, is

$$
G_{0}^{R, A}\left(\mathbf{r}_{i}, \mathbf{r}, k_{0}\right)=-\frac{1}{4 \pi} \frac{e^{ \pm i k_{0} R}}{R}
$$

\subsection{Electromagnetic waves}

The formalism of Green functions provides an appropriate technical framework for the study of solutions of wave equations for free systems namely without sources. It becomes essential for the study of the propagation of electromagnetic waves from a distribution of sources $j(\mathbf{r})$ of the field. This is the problem of radiative transfer [2]. For a pointlike source, we are back to the previous problem of the Green equation. In the general case, The Helmholtz equation (2) needs to be replaced by

$$
\Delta \psi(\mathbf{r})+k_{0}^{2}(1+\mu(\mathbf{r})) \psi(\mathbf{r})=j(\mathbf{r})
$$

where $\psi(\mathbf{r})$ is indeed a Green function i.e. it depends on the distribution of sources $j(\mathbf{r})$. The equation (13) can also be written in the form of the integral equation

$$
\psi(\mathbf{r})=\int d \mathbf{r}_{i} j\left(\mathbf{r}_{i}\right) G_{0}\left(\mathbf{r}_{i}, \mathbf{r}, k_{0}\right)-k_{0}^{2} \int d \mathbf{r}^{\prime} \psi\left(\mathbf{r}^{\prime}\right) \mu\left(\mathbf{r}^{\prime}\right) G_{0}\left(\mathbf{r}^{\prime}, \mathbf{r}, k_{0}\right)
$$

which allows to consider separately the effects of the source and of the random potential $\mu(\mathbf{r})$.

\section{Multiple scattering expansion}

Either the expressions (9) or (14) provide the starting point for a systematic expansion of the Green function in terms of the free Green function. It can be written

$$
\begin{aligned}
G\left(\mathbf{r}, \mathbf{r}^{\prime}\right) & =G_{0}\left(\mathbf{r}, \mathbf{r}^{\prime}\right)+\int d \mathbf{r}_{1} G_{0}\left(\mathbf{r}, \mathbf{r}_{1}\right) V\left(\mathbf{r}_{1}\right) G_{0}\left(\mathbf{r}_{1}, \mathbf{r}^{\prime}\right) \\
& +\int d \mathbf{r}_{1} d \mathbf{r}_{2} G_{0}\left(\mathbf{r}, \mathbf{r}_{1}\right) V\left(\mathbf{r}_{1}\right) G_{0}\left(\mathbf{r}_{1}, \mathbf{r}_{2}\right) V\left(\mathbf{r}_{2}\right) G_{0}\left(\mathbf{r}_{2}, \mathbf{r}^{\prime}\right)+\ldots
\end{aligned}
$$


We can now calculate the average Green function using the white noise potential (位). All the odd terms in the potential $V$ disappear from (15), and it remains

$$
\bar{G}\left(\mathbf{r}, \mathbf{r}^{\prime}\right)=G_{0}\left(\mathbf{r}, \mathbf{r}^{\prime}\right)+\gamma \int d \mathbf{r}_{1} G_{0}\left(\mathbf{r}, \mathbf{r}_{1}\right) G_{0}\left(\mathbf{r}_{1}, \mathbf{r}_{1}\right) G_{0}\left(\mathbf{r}_{1}, \mathbf{r}^{\prime}\right)+\ldots
$$

where we denote from now on the disorder average by $\ldots$. By averaging over the disorder, the medium becomes again translational invariant and the Green function $\bar{G}\left(\mathbf{r}, \mathbf{r}^{\prime}\right)=\bar{G}\left(\mathbf{r}-\mathbf{r}^{\prime}\right)$. The average over the disorder generates all possible diagrams. Among them, there is a subclass called irreducible diagrams that cannot be split into two already existing diagrams without cutting an impurity line. It is possible to rewrite the average Green function or its Fourier transform in terms of the contribution of these diagrams only. We then obtain the so called Dyson equation

$$
\bar{G}(\mathbf{k})=G_{0}(\mathbf{k})+G_{0}(\mathbf{k}) \Sigma(\mathbf{k}, \epsilon) \bar{G}(\mathbf{k})
$$

where the function $\Sigma(\mathbf{k}, \epsilon)$ is called the self-energy. It should be emphasized that, although the selfenergy contains only the irreducible diagrams, there is an infinity of them. Thus, the calculation of $\Sigma$ is a difficult problem. For the white noise potential (画, $\Sigma$ can be expanded in powers of $\gamma$. To first order, for the Schrödinger equation, we obtain

$$
\Sigma_{1}^{R, A}(\mathbf{k}, \epsilon)=\frac{\gamma}{\Omega} \sum_{\mathbf{q}} G_{0}^{R, A}(\mathbf{q})
$$

where $\Omega$ is the volume of the system. The real part of $\Sigma$ corresponds to an irrelevant shift of the origin of the energies that we shall ignore. The imaginary part is

$$
\operatorname{Im} \Sigma_{1}^{R}(\mathbf{k}, \epsilon)=-\pi \rho_{0}(\epsilon) \gamma
$$

while for the Helmholtz equation, it is

$$
\operatorname{Im} \Sigma_{1}^{R}(\mathbf{k}, \epsilon)=-\frac{\gamma k_{0}}{4 \pi}
$$

We emphasize again that the difference between these two expressions results from the two distinct dispersions of respectively the Schrödinger and Helmholtz equations. Higher orders terms in the expansion of $\Sigma$ are proportional to $\operatorname{Im} \Sigma_{1}^{R}$ times some power of the dimensionless parameter $\frac{1}{k l_{e}}$. The contribution $\Sigma_{1}$ describes the multiple scattering of the wave as a series of independent effective collisions. The higher corrections include interference effects between those successive scattering events. The weak disorder limit $k l_{e} \gg 1$ amounts to neglecting these interferences. We deal then with the so called self-consistent Born approximation. We shall, from now on, consider only this limit.

It is then a straightforward calculation to get an expression for the average Green function at this approximation:

$$
\bar{G}^{R, A}\left(\mathbf{r}_{i}, \mathbf{r}, k_{0}\right)=G_{0}^{R, A}\left(\mathbf{r}_{i}, \mathbf{r}, k_{0}\right) e^{-\left|\mathbf{r}-\mathbf{r}_{i}\right| / 2 l_{e}}
$$

To conclude this section, we would like to notice that although this expression has been obtained for the case of an infinite system, this restrictive assumption can be released and we need to consider, for this relation to be valid, only systems of sizes $L \gg l_{e}$.

\section{$5 \quad$ Probability of quantum diffusion}

The quantities of physical interest are usually not related to the average Green function but instead to the so called probability of quantum diffusion which describes the probability for a quantum particle (or a wave) to go from the point $\mathbf{r}$ to the point $\mathbf{r}^{\prime}$ in a time $t$. Once we average over the disorder, we shall see that this probability $P\left(\mathbf{r}, \mathbf{r}^{\prime}, t\right)$ contains mainly three contributions:

- i. The probability to go from $\mathbf{r}$ to $\mathbf{r}^{\prime}$ without scattering. 
- ii. The probability to go from $\mathbf{r}$ to $\mathbf{r}^{\prime}$ by an incoherent sequence of multiple scattering, which is called the diffuson.

- iii. The probability to go from $\mathbf{r}$ to $\mathbf{r}^{\prime}$ by a coherent multiple scattering sequence. We shall calculate one such coherent process, called the cooperon.

We shall first define the probability for the Schrödinger case. Please notice that througout this section we shall take $\hbar=1$. To that purpose, we consider a gaussian wavepacket of energy $\epsilon_{0}$. We shall also assume that around $\epsilon_{0}$ the density of states is constant. Then, we can write for the Fourier transform $P\left(\mathbf{r}, \mathbf{r}^{\prime}, \omega\right)$ of the probability $P\left(\mathbf{r}, \mathbf{r}^{\prime}, t\right)$ the expression:

$$
P\left(\mathbf{r}, \mathbf{r}^{\prime}, \omega\right)=\frac{1}{2 \pi \rho_{0}} \overline{G^{R}\left(\mathbf{r}, \mathbf{r}^{\prime}, \epsilon_{0}\right) G^{A}\left(\mathbf{r}^{\prime}, \mathbf{r}, \epsilon_{0}-\omega\right)}
$$

This probability is normalized to unity which means that either

$$
\int P\left(\mathbf{r}, \mathbf{r}^{\prime}, t\right) d \mathbf{r}^{\prime}=1
$$

or

$$
\int P\left(\mathbf{r}, \mathbf{r}^{\prime}, \omega\right) d \mathbf{r}^{\prime}=\frac{i}{\omega}
$$

\subsection{Free propagation}

In the absence of disorder, the Green functions in (22) take their free expression (10) and it is straightforward to obtain for the three dimensional case

$$
P\left(\mathbf{r}, \mathbf{r}^{\prime}, t\right)=\frac{\delta(R-v t)}{4 \pi R^{2}}
$$

where $R=\left|\mathbf{r}^{\prime}-\mathbf{r}\right|$ and $v$ being the group velocity. This probability is indeed normalized.

\subsection{Drude-Boltzmann approximation}

In the presence of disorder, we need, in order to calculate the probability, to evaluate the average of the product of the two Green functions that appear in (22). The simplest approximation is to replace the average by the product of the two averaged Green functions. Here again, since we have calculated in the weak disorder limit (21) the expression of $\bar{G}$, we obtain

$$
P_{0}\left(\mathbf{r}, \mathbf{r}^{\prime}, \omega\right)=\frac{e^{i \omega R / v-R / l_{e}}}{4 \pi R^{2} v}
$$

so that

$$
\int P_{0}\left(\mathbf{r}, \mathbf{r}^{\prime}, \omega\right) d \mathbf{r}^{\prime}=\frac{\tau_{e}}{1-i \omega \tau_{e}}
$$

At this approximation, the probability is not normalized, but instead

$$
\int P_{0}\left(\mathbf{r}, \mathbf{r}^{\prime}, t\right) d \mathbf{r}^{\prime}=e^{-t / \tau_{e}}
$$

It is then clear that some part is missing in the probability. The Drude-Boltzmann approximation overlooks a large part of the probability; since after a time $t$, it predicts that the wavepacket disappears. 


\subsection{The diffuson}

There is another contribution associated to the multiple scattering which can be calculated in the weak disorder limit $k l_{e} \gg 1$ in a semiclassical way. Using the description we obtained previously for the calculation of the average Green function, we can associate [3] to each possible sequence $\mathcal{C}$, of independent effective collisions a complex amplitude $A\left(\mathbf{r}, \mathbf{r}^{\prime}, \mathcal{C}\right)$. Then, using a generalization of the Feynman path integral description, we can in principle write the Green function as a sum of such complex amplitudes.

Then, in order to evaluate the product of two Green functions, we notice the following two points.

- i. Due to the short range of the scattering potential, the set of scatterers entering in the sequences for both $G^{R}$ and $G^{A}$ must be identical.

- ii. For the effective collisions, the mean distance between them is set by the elastic mean free path $l_{e} \gg \lambda$. Therefore, if any two scattering sequences differ by even one collision event, the phase difference between the two complex amplitudes, which measures the difference of path lengths in units of $\lambda$ will be very large and then the corresponding probability will vanish on average.

We shall therefore retain only contributions for which the corresponding probability $P_{d}\left(\mathbf{r}, \mathbf{r}^{\prime}, \omega\right)$ is

$$
\begin{aligned}
P_{d}\left(\mathbf{r}, \mathbf{r}^{\prime}, \omega\right) & =\frac{1}{2 \pi \rho_{0}} \int \bar{G}_{\epsilon}^{R}\left(\mathbf{r}, \mathbf{r}_{1}\right) \bar{G}_{\epsilon-\omega}^{A}\left(\mathbf{r}_{1}, \mathbf{r}\right) \bar{G}_{\epsilon}^{R}\left(\mathbf{r}_{2}, \mathbf{r}^{\prime}\right) \bar{G}_{\epsilon-\omega}^{A}\left(\mathbf{r}^{\prime}, \mathbf{r}_{2}\right) \times \\
& \times \Gamma_{\omega}\left(\mathbf{r}_{1}, \mathbf{r}_{2}\right) d \mathbf{r}_{1} d \mathbf{r}_{2}
\end{aligned}
$$

It is made of two multiplicative contributions. The first one is

$$
\bar{G}_{\epsilon}^{R}\left(\mathbf{r}, \mathbf{r}_{1}\right) \bar{G}_{\epsilon}^{R}\left(\mathbf{r}_{2}, \mathbf{r}^{\prime}\right) \bar{G}_{\epsilon-\omega}^{A}\left(\mathbf{r}_{1}, \mathbf{r}\right) \bar{G}_{\epsilon-\omega}^{A}\left(\mathbf{r}^{\prime}, \mathbf{r}_{2}\right) .
$$

It describes the mean propagation between whatever two points $\mathbf{r}$ and $\mathbf{r}^{\prime}$ in the medium and the first $\left(\mathbf{r}_{1}\right)$ (respectively the last $\left(\mathbf{r}_{2}\right)$ ) collision event of the sequence of scattering events. The second contribution defines the quantity $\Gamma_{\omega}\left(\mathbf{r}_{1}, \mathbf{r}_{2}\right)$ which we shall call the structure factor of the scattering medium. In a sense, it generalizes to the multiple scattering situation the usual twopoint correlation function in the single scattering case. We now use once again the assumption of independent collisions in order to write for $\Gamma_{\omega}\left(\mathbf{r}_{1}, \mathbf{r}_{2}\right)$ the integral equation

$$
\Gamma_{\omega}\left(\mathbf{r}_{1}, \mathbf{r}_{2}\right)=\gamma \delta\left(\mathbf{r}_{1}-\mathbf{r}_{2}\right)+\gamma \int \bar{G}_{\epsilon}^{R}\left(\mathbf{r}_{1}, \mathbf{r}\right) \bar{G}_{\epsilon-\omega}^{A}\left(\mathbf{r}, \mathbf{r}_{1}\right) \Gamma_{\omega}\left(\mathbf{r}, \mathbf{r}_{2}\right) d \mathbf{r}
$$

This equation can be solved exactly in some geometries. For the infinite three dimensional space, we can make use of the translational invariance and get for the structure factor the expression

$$
\Gamma_{\omega}(\mathbf{q})=\frac{\gamma}{1-P_{0}(\mathbf{q}, \omega) / \tau_{e}}
$$

where $P_{0}(\mathbf{q}, \omega)$ is the Fourier transform of (26) and is given by $\frac{1}{q v} \arctan \frac{q l_{e}}{1-i \omega \tau_{e}}$ with $q=|\mathbf{q}|$. Then, the probability rewrites

$$
P_{d}(\mathbf{q}, \omega)=P_{0}(\mathbf{q}, \omega) \frac{P_{0}(\mathbf{q}, \omega) / \tau_{e}}{1-P_{0}(\mathbf{q}, \omega) / \tau_{e}}
$$

Using this expression of $P_{d}$, the normalization of the total probability $P=P_{0}+P_{d}$ can be readily checked namely $P(\mathbf{q}=0, \omega)=\frac{i}{\omega}$. For the semi-infinite space with a point source, it is also possible to obtain a closed analytical expression for the probability $P_{d}$ using the Wiener-Hopf method. But beyond these two cases, for simple finite geometries, it is not possible to obtain the solution of (29) without resorting to numerical calculations. We are then led to look for some approximate 
solutions. An excellent one is the diffusion approximation obtained for large times $t \gg \tau_{e}$ and large spatial variations $r \gg l_{e}$. It is obtained by expanding the structure factor under the form

$$
\Gamma_{\omega}\left(\mathbf{r}, \mathbf{r}_{2}\right)=\Gamma_{\omega}\left(\mathbf{r}_{1}, \mathbf{r}_{2}\right)+\left(\mathbf{r}-\mathbf{r}_{1}\right) \cdot \nabla_{\mathbf{r}_{1}} \Gamma_{\omega}+\frac{1}{2}\left[\left(\mathbf{r}-\mathbf{r}_{1}\right) \cdot \nabla_{\mathbf{r}_{1}}\right]^{2} \Gamma_{\omega}
$$

which together with the integral equation (30) gives

$$
\left[-i \omega-D \Delta_{\mathbf{r}_{1}}\right] \Gamma_{\omega}\left(\mathbf{r}_{1}, \mathbf{r}_{2}\right)=\frac{1}{2 \pi \rho_{0} \tau_{e}^{2}} \delta\left(\mathbf{r}_{1}-\mathbf{r}_{2}\right)
$$

where the diffusion coefficient is $D=\frac{1}{d} \frac{l_{e}^{2}}{\tau_{e}}=\frac{1}{d} v^{2} \tau_{e}$. At this approximation, we have between $P_{d}$ and $\Gamma_{\omega}$ the following relation

$$
P_{d}\left(\mathbf{r}, \mathbf{r}^{\prime}, \omega\right) \simeq 2 \pi \rho_{0} \tau_{e}^{2} \Gamma_{\omega}\left(\mathbf{r}, \mathbf{r}^{\prime}\right)
$$

so that $P_{d}$, as well, obeys a diffusion equation. It is interesting to check the validity of the diffusion approximation. For an infinite system, and for $\frac{r}{l_{e}}=1$ the relative correction between the exact solution and diffusion approximation is 0.085 while for $\frac{r}{l_{e}}=2.5$, it is less than $5.10^{-3}$.

\subsection{The cooperon}

With the normalized expression of the probability we just have obtained, it seems that we fulfilled the demand of evaluating all the relevant processes that contribute to the probability. But it could be, and it is certainly the case, that there are many other contributions that sum up to zero.

For instance, we may consider the possibility which corresponds to the product of Green functions such as we considered before, but where now the two identical trajectories are time reversed one from the other. It is clear that if these trajectories are closed on themselves, there is no phase difference left between them. This requires that the system has time-reversal invariance namely that $G^{R, A}\left(\mathbf{r}, \mathbf{r}^{\prime}, t\right)=G^{R, A}\left(\mathbf{r}^{\prime}, \mathbf{r}, t\right)$. This relation does not hold anymore in the presence of a magnetic field for electronic systems.

The contribution, we shall call $P_{c}$, of this process to the total probability can be evaluated as we did before for the diffuson. Thus we have instead of (29)

$$
\begin{aligned}
P_{c}\left(\mathbf{r}, \mathbf{r}^{\prime}, \omega\right) & =\frac{1}{2 \pi \rho_{0}} \int \bar{G}_{\epsilon}^{R}\left(\mathbf{r}, \mathbf{r}_{1}\right) \bar{G}_{\epsilon}^{R}\left(\mathbf{r}_{2}, \mathbf{r}^{\prime}\right) \bar{G}_{\epsilon-\omega}^{A}\left(\mathbf{r}^{\prime}, \mathbf{r}_{1}\right) \bar{G}_{\epsilon-\omega}^{A}\left(\mathbf{r}_{2}, \mathbf{r}\right) \times \\
& \times \Gamma_{\omega}^{\prime}\left(\mathbf{r}_{1}, \mathbf{r}_{2}\right) d \mathbf{r}_{1} d \mathbf{r}_{2}
\end{aligned}
$$

where the new structure factor $\Gamma_{\omega}^{\prime}$ is solution of the integral equation

$$
\Gamma_{\omega}^{\prime}\left(\mathbf{r}_{1}, \mathbf{r}_{2}\right)=\gamma \delta\left(\mathbf{r}_{1}-\mathbf{r}_{2}\right)+\gamma \int \bar{G}_{\epsilon}^{R}\left(\mathbf{r}_{1}, \mathbf{r}^{\prime \prime}\right) \bar{G}_{\epsilon-\omega}^{A}\left(\mathbf{r}_{1}, \mathbf{r}^{\prime \prime}\right) \Gamma_{\omega}^{\prime}\left(\mathbf{r}^{\prime \prime}, \mathbf{r}_{2}\right) d \mathbf{r}^{\prime \prime}
$$

Notice that unlike the structure factor of the diffuson, the new combination $\bar{G}_{\epsilon}^{R}\left(\mathbf{r}_{1}, \mathbf{r}^{\prime \prime}\right) \bar{G}_{\epsilon-\omega}^{A}\left(\mathbf{r}_{1}, \mathbf{r}^{\prime \prime}\right)$ cannot be simply written in terms of the probability $P_{0}$. But as before, we can evaluate $P_{c}\left(\mathbf{r}, \mathbf{r}^{\prime}, \omega\right)$ in the diffusion approximation (i.e. for slow variations) and we obtain

$$
P_{c}\left(\mathbf{r}, \mathbf{r}^{\prime}, \omega\right) \simeq \frac{\Gamma_{\omega}(\mathbf{r}, \mathbf{r})}{2 \pi \rho_{0}}\left[\int \bar{G}_{\epsilon}^{R}\left(\mathbf{r}, \mathbf{r}_{1}\right) \bar{G}_{\epsilon}^{A}\left(\mathbf{r}^{\prime}, \mathbf{r}_{1}\right) d \mathbf{r}_{1}\right]^{2}
$$

Since in the presence of time reversal invariance, we have

$$
\bar{G}_{\epsilon}^{R}\left(\mathbf{r}_{1}, \mathbf{r}\right) \bar{G}_{\epsilon}^{A}\left(\mathbf{r}_{1}, \mathbf{r}\right)=\bar{G}_{\epsilon}^{R}\left(\mathbf{r}_{1}, \mathbf{r}\right) \bar{G}_{\epsilon}^{A}\left(\mathbf{r}, \mathbf{r}_{1}\right)
$$

then, $\Gamma_{\omega}^{\prime}\left(\mathbf{r}_{1}, \mathbf{r}_{2}\right)=\Gamma_{\omega}\left(\mathbf{r}_{1}, \mathbf{r}_{2}\right)$ and finally,

$$
P_{c}\left(\mathbf{r}, \mathbf{r}^{\prime}, \omega\right)=P_{d}(\mathbf{r}, \mathbf{r}, \omega) g\left(\mathbf{r}-\mathbf{r}^{\prime}\right)^{2}
$$


where in $3 d$ we have the relation

$$
g(R)=\frac{\sin k R}{k R} e^{-R / 2 l_{e}}
$$

and $R=\left|\mathbf{r}-\mathbf{r}^{\prime}\right|$. For $R=0$, i.e. for $\mathbf{r}=\mathbf{r}^{\prime}$, we have

$$
P_{c}(\mathbf{r}, \mathbf{r}, \omega)=P_{d}(\mathbf{r}, \mathbf{r}, \omega)
$$

namely, the probability to come back to the initial point is twice the value given by the diffuson. The contribution of the cooperon $P_{c}$ to the total probability is given by

$$
\int P_{c}\left(\mathbf{r}, \mathbf{r}^{\prime}, \omega\right) d \mathbf{r}^{\prime}=P_{d}(\mathbf{r}, \mathbf{r}, \omega) \frac{\tau_{e}}{\pi \rho_{0}}
$$

for any space dimensionality. How does this contribution compare with the diffuson contribution? We have found that $P_{d}(q=0, t) \simeq 1$ while $P_{c}(q=0, t) \simeq \frac{\tau_{e}}{\pi \rho_{0}} \frac{1}{(D t)^{3 / 2}}$ for small enough times. Then, $P_{c}(q=0, t)$ is maximum for $t \simeq \tau_{e}$ and given by $P_{c}\left(q=0, \tau_{e}\right)=\frac{1}{\left(k l_{e}\right)^{d-1}}$. Thus, the contribution of $P_{c}$ to the total probability is vanishingly small for $k l_{e} \gg 1$ and for a space dimensionality $d \geq 2$. But although it is very small, $P_{c}$ must be compensated by another contribution in order to restore the normalization of the probability. The additional contributions result from other irreducible diagrams. But the subsequent terms in this series are not known.

We would like to conclude this section on the cooperon by emphasizing that although $\Gamma_{\omega}^{\prime}$ obeys a diffusion equation, it would be meaningless and incorrect to state that $P_{c}\left(\mathbf{r}, \mathbf{r}^{\prime}, \omega\right)$ obeys it as well. The exact statement is that for $\mathbf{r}=\mathbf{r}^{\prime}, P_{c}$ and $P_{d}$ are proportional and that $P_{d}$ obeys a diffusion equation.

\section{Radiative transfer}

\subsection{Local intensity and correlation function}

In the previous sections, we have defined the quantum probability for electronic systems. The probability $P$ is directly related to quantities that are physically measurable like the electrical conductivity or the magnetic response e.g. the magnetization [4, 5]. For the study of the propagation of electromagnetic waves in disordered media, the quantity which is usually measured is the local intensity of the field or its correlation function [6]. As we discussed previously, and by definition of the Green function, the radiative solution $\psi_{\epsilon}(\mathbf{r})$ of the Helmholtz equation (13) with a localized source at point $\mathbf{R}=\mathbf{0}$ is $\bar{\psi}_{\epsilon}(\mathbf{r})=G_{\epsilon}(\mathbf{0}, \mathbf{r})$. The correlation function of the field is then

$$
\overline{\psi_{\epsilon}(\mathbf{r}) \psi_{\epsilon-\omega}^{*}\left(\mathbf{r}^{\prime}\right)}=\overline{G_{\epsilon}^{R}(\mathbf{0}, \mathbf{r}) G_{\epsilon-\omega}^{A}\left(\mathbf{r}^{\prime}, \mathbf{0}\right)}
$$

It is not directly related to the probability of quantum diffusion $P\left(\mathbf{r}, \mathbf{r}^{\prime}, \omega\right)$. But the radiated intensity $I(\mathbf{r})$ defined by

$$
\begin{aligned}
I(\mathbf{r}) & =\frac{4 \pi}{c}\left|\psi_{\epsilon}(\mathbf{r})\right|^{2} \\
& =\frac{4 \pi}{c} G_{\epsilon}^{R}(\mathbf{0}, \mathbf{r}) G_{\epsilon}^{A}(\mathbf{r}, \mathbf{0})
\end{aligned}
$$

is indeed related on average to the probability $P$ and

$$
\bar{I}(\mathbf{r})=\frac{4 \pi}{c} \overline{G_{\epsilon}^{R}(\mathbf{0}, \mathbf{r}) G_{\epsilon}^{A}(\mathbf{r}, \mathbf{0})}
$$

Using for the probability the following relation which is the counterpart, for the Helmholtz equation, of the relation (22)

$$
P_{d}\left(\mathbf{r}, \mathbf{r}^{\prime}\right)=\frac{4 \pi}{c} \overline{G_{\epsilon}^{R}\left(\mathbf{r}, \mathbf{r}^{\prime}\right) G_{\epsilon}^{A}\left(\mathbf{r}^{\prime}, \mathbf{r}\right)}
$$


we obtain $\bar{I}(\mathbf{r})=P_{d}(\mathbf{0}, \mathbf{r})$. From now on, we shall denote $I(\mathbf{r})$ the average intensity.

We can rephrase what we did before in order to calculate the various contributions to the intensity that come respectively from the Drude-Boltzman, the diffuson and the cooperon approximations. The first contribution is given by

$$
I_{0}(R)=\frac{1}{4 \pi R^{2} c} e^{-R / l_{e}}
$$

It corresponds to the contribution to the radiative intensity of waves that did not experience any collision on a distance $R$ from the source.

The diffuson contribution is given by

$$
I_{d}(\mathbf{r})=\frac{4 \pi}{c} \int d \mathbf{r}_{1} d \mathbf{r}_{2}\left|\bar{\psi}_{\epsilon}\left(\mathbf{r}_{1}\right)\right|^{2} \Gamma_{\omega=0}\left(\mathbf{r}_{1}, \mathbf{r}_{2}\right)\left|\bar{G}_{\epsilon}^{R}\left(\mathbf{r}_{2}, \mathbf{r}\right)\right|^{2}
$$

and finally, the contribution of the cooperon to the intensity is

$$
I_{c}(\mathbf{r})=\frac{4 \pi}{c} \int d \mathbf{r}_{1} d \mathbf{r}_{2} \bar{\psi}_{\epsilon}\left(\mathbf{r}_{1}\right) \bar{\psi}_{\epsilon}^{*}\left(\mathbf{r}_{2}\right) \Gamma\left(\mathbf{r}_{1}, \mathbf{r}_{2}\right) \bar{G}_{\epsilon}^{R}\left(\mathbf{r}_{2}, \mathbf{r}\right) \bar{G}_{\epsilon}^{A}\left(\mathbf{r}, \mathbf{r}_{1}\right)
$$

where we used the notation $\Gamma_{\omega=0}=\Gamma$. In the diffuson approximation, the intensity $I_{d}$ rewrites

$$
I_{d}(\mathbf{r})=P_{d}(\mathbf{0}, \mathbf{r})=\frac{l_{e}^{2}}{4 \pi c} \Gamma(\mathbf{0}, \mathbf{r})
$$

and like $P_{d}$, it obeys the diffuson equation

$$
-D \Delta I_{d}(\mathbf{r})=\delta(\mathbf{r})
$$

whose solution in the $3 d$ free space is

$$
I_{d}(R)=\frac{1}{4 \pi D R}
$$

We shall now apply all the considerations developed in this section to the calculations of physical quantities in some specific situations both for metallic systems and for the propagation of electromagnetic waves in suspensions.

\section{Example 1 . The electrical conductivity of a weakly dis- ordered metal}

We previously defined a weakly disordered metal as a non interacting and degenerate (spinless) electron gas (at $T=0$ ), moving in the field of defects and impurities described by the white noise potential (14).

The average electrical conductivity $\sigma(\omega)$ calculated in the framework of the linear response theory [7] is given by the Kubo formula:

$$
\sigma(\omega)=\frac{e^{2} \hbar^{3}}{2 \pi m^{2} \Omega} \sum_{\mathbf{k}, \mathbf{k}^{\prime}} k_{x} k_{x}^{\prime} \overline{G_{\epsilon}^{R}\left(\mathbf{k}, \mathbf{k}^{\prime}\right) G_{\epsilon-\omega}^{A}\left(\mathbf{k}^{\prime}, \mathbf{k}\right)}
$$

Then, we see from this definition, that the structure of the conductivity is up to the product $k_{x} k_{x}^{\prime}$ very similar to those of the quantum probability $P$. Therefore, and just as we did before, the very definition of $\sigma(\omega)$ leads us to the following set of approximations. 


\subsection{The Drude-Boltzman approximation}

It is obtained by replacing the average of the product of two Green functions by the product of the averages, namely

$$
\overline{G_{\epsilon}^{R}\left(\mathbf{k}, \mathbf{k}^{\prime}\right) G_{\epsilon-\omega}^{A}\left(\mathbf{k}^{\prime}, \mathbf{k}\right)} \simeq \bar{G}_{\epsilon}^{R}\left(\mathbf{k}, \mathbf{k}^{\prime}\right) \bar{G}_{\epsilon-\omega}^{A}\left(\mathbf{k}^{\prime}, \mathbf{k}\right)
$$

where the Fourier transform of the averaged Green functions (21) is

$$
\bar{G}_{\epsilon}^{R, A}\left(\mathbf{k}, \mathbf{k}^{\prime}\right)=\bar{G}_{\epsilon}^{R, A}(\mathbf{k}) \delta_{\mathbf{k}, \mathbf{k}^{\prime}}=\frac{\delta_{\mathbf{k}, \mathbf{k}^{\prime}}}{\epsilon-\epsilon(\mathbf{k}) \pm i \frac{\hbar}{2 \tau_{e}}}
$$

Then, we obtain for the conductivity $\sigma_{0}(\omega)$ at this approximation the following expression

$$
\sigma_{0}(\omega)=\frac{n e^{2}}{m} \int d \mathbf{r}^{\prime} P_{0}\left(\mathbf{r}, \mathbf{r}^{\prime}, \omega\right)
$$

where $P_{0}$ is the quantum probability calculated at the same approximation (26). Using the expression (27), we obtain

$$
\sigma_{0}(\omega)=\frac{n e^{2}}{m} \frac{\tau_{e}}{1-i \omega \tau_{e}}
$$

which is the well-known Drude expression. It must be noticed that because of the Kronecker delta function that appears in the average Green functions, the scalar product $k_{x} k_{x}^{\prime}$ reduces simply to $k_{x}^{2}$ and eventually, after averaging, to $\frac{k_{F}^{2}}{d}$ where $k_{F}$ is the Fermi wavevector.

\subsection{The contributions of the diffuson and the cooperon}

Here again, we approximate the average product in the relation (54), using the same scheme we used for the diffuson. The contribution $\sigma_{d}(\omega)$ of the diffuson to the conductivity is thus

$$
\sigma_{d}(\omega)=\frac{e^{2} \hbar^{3}}{2 \pi m^{2} \Omega^{2}} \frac{1}{2 \pi \rho_{0} \tau_{e}^{2}} P_{d}(\mathbf{0}, \omega) \sum_{\mathbf{k}, \mathbf{k}^{\prime}} k_{x} k_{x}^{\prime} \tilde{P}_{0}(\mathbf{k}, \mathbf{0}, \omega) \tilde{P}_{0}\left(\mathbf{k}^{\prime}, \mathbf{0}, \omega\right)
$$

where the function $\tilde{P}_{0}(\mathbf{k}, \mathbf{q}, \omega)=\frac{1}{2 \pi \rho_{0}} \bar{G}_{\epsilon}^{R}\left(\mathbf{k}+\frac{\mathbf{q}}{2}\right) \bar{G}_{\epsilon-\omega}^{A}\left(\mathbf{k}-\frac{\mathbf{q}}{2}\right)$. Since the function $\tilde{P}_{0}(\mathbf{k}, \mathbf{q}=\mathbf{0}, \omega)$ depends only on the modulus of the wavevector $\mathbf{k}$ and not on his direction, the angular integral in the previous expression gives a vanishing contribution namely $\sigma_{d}(\omega)=0$. Then, it is interesting to notice that although the diffuson gives the main contribution to the quantum probability, its contribution to the conductivity which indeed measures such a probability, vanishes identically.

We evaluate, the contribution $\sigma_{c}(\omega)$ of the cooperon using the relation (36) so that

$$
\sigma_{c}(\omega)=-\frac{e^{2} \hbar^{3}}{2 \pi m^{2}} \frac{k_{F}^{2}}{d}\left[\frac{1}{\Omega} \sum_{\mathbf{k}} \tilde{P}_{0}^{2}(k, \mathbf{q}=\mathbf{0}, \omega)\right] \frac{1}{\Omega} \sum_{\mathbf{Q}} P_{d}(\mathbf{Q}, \omega)
$$

the sum in the brackets is straightforward so that in the diffusion approximation, we obtain

$$
\sigma_{c}(\omega)=-\frac{e^{2} D}{\pi \hbar} P_{d}(\mathbf{r}, \mathbf{r}, \omega)
$$

Using now the relation (43) between the cooperon and the diffuson, $\sigma_{c}(\omega)$ rewrites

$$
\sigma_{c}(\omega)=-\frac{n e^{2}}{m} \int d \mathbf{r}^{\prime} P_{c}\left(\mathbf{r}, \mathbf{r}^{\prime}, \omega\right)
$$

and the total conductivity at this order is now given by

$$
\sigma(\omega)=\frac{n e^{2}}{m} \int d \mathbf{r}^{\prime}\left(P_{0}\left(\mathbf{r}, \mathbf{r}^{\prime}, \omega\right)-P_{c}\left(\mathbf{r}, \mathbf{r}^{\prime}, \omega\right)\right)
$$

The conductivity $\sigma(\omega)$ is reduced by the coherent (cooperon) contribution. This correction is called the weak localization correction to the conductivity [8, 9, 10]. Relatively, this contribution of $P_{c}$ is much larger than the normalization correction to the total probability. This is because $P_{c}$ is now compared to $P_{0}$ and not to $P_{d}$ which represents the main contribution. 


\subsection{The recurrence time}

It is of some interest to study the dc conductivity $\sigma(\omega=0)$ using a slightly different point of view [1]. ¿From the relation (63), and using the dc expression $\sigma_{0}=\frac{n e^{2} \tau_{e}}{m}$, we obtain for the relative correction to the conductivity,

$$
\frac{\delta \sigma}{\sigma_{0}}=-\frac{1}{\pi \hbar \rho_{0}} \int_{0}^{\infty} d t P_{c}(\mathbf{r}, \mathbf{r}, t)
$$

where $\rho_{0}$ is the density of states per unit volume.

Consider now the quantity $Z(t)$ defined by

$$
Z(t)=\int_{\Omega} P_{d}(\mathbf{r}, \mathbf{r}, t) d \mathbf{r}
$$

where the integral is over the volume $\Omega$ of the system. It represents the return probability to a point $\mathbf{r}$ averaged over all those points. This quantity which characterizes the solutions of the diffusion equation is sometimes called the heat kernel in the literature. The time integral of $Z(t)$ defines the characteristic time $T_{R}$

$$
T_{R}=\int_{\Omega} d \mathbf{r} \int_{0}^{\infty} P_{d}(\mathbf{r}, \mathbf{r}, t) d t=\int_{0}^{\infty} Z(t) d t
$$

called the recurrence time. It measures the space average of the time spent by a diffusive particle within each infinitesimal volume. $T_{R}$ diverges, as stated by the Polya theorem [1], for a random walk in the free space of dimensionality $d \leq 2$. Then, we define a regularized expression for $T_{R}$ given by the Laplace transform

$$
T_{R}(s)=\int_{0}^{\infty} Z(t) e^{-s t} d t
$$

This expression of the recurrence time may be interpreted by saying that $T_{R}(s)$ selects the contribution of all the diffusive trajectories of lengths smaller than $L_{s}=\sqrt{D / s}$.

Then, the expression (64) of the relative contribution of the cooperon to the conductivity rewrites

$$
\frac{\delta \sigma}{\sigma_{0}}=-\frac{\Delta}{\pi \hbar} \int_{0}^{\infty} d t Z(t) e^{-s t}=-\frac{\Delta}{\pi \hbar} T_{R}(s)
$$

where the energy defined by $\Delta=\frac{1}{\Omega \rho_{0}}$ is the mean level spacing measured at the Fermi level between the energy levels of an electron gas confined in a box of volume $\Omega$. The weak localization correction to the dc conductivity can be essentially expressed in terms of a purely classical quantity, namely the recurrence time $T_{R}$. When it diverges, we need to use its regularized version (67), where now the length $L_{s}$ can be given a physical meaning. The cooperon correction results from the absence of any relative phase between two time reversed multiple scattering trajectories. Any interaction of an electron with an external perturbation may destroy this phase coherence and then the contribution of the cooperon. For a large class of such perturbations which includes inelastic collisions at finite temperature, Coulomb interactions or coupling to other excitations, we can define a phenomenological length $L_{\phi}$ called the phase coherence length and a phase coherence time $\tau_{\phi}$ such that $L_{\phi}^{2}=D \tau_{\phi}$. Thus, $L_{\phi}$ is the length $L_{s}$ used to regularize the recurrence time. We shall see later other examples of physical quantities that can be expressed using either the heat kernel or the recurrence time.

\subsection{Conductance fluctuations- Spectral determinant}

We could as well calculate the so-called conductance fluctuations using the properties of the heat kernel. The conductance $G$ is related to the conductivity $\sigma$ by the Ohm's law $G=\sigma L^{d-2}$. We define the dimensionless conductance $g=\frac{h}{e^{2}} G$. Its fluctuation defined by $\left\langle\delta g^{2}\right\rangle=\left\langle g^{2}\right\rangle-\langle g\rangle^{2}$ can be expressed in terms of $Z(t)$ through

$$
\left\langle\delta g^{2}\right\rangle=\frac{12}{\tau_{D}^{2}} \int_{0}^{\infty} d t t Z(t) e^{-s t}
$$


where $\tau_{D}=L^{2} / D$ is the diffusion time. For a one dimensional system (in the sense of the diffusion equation), we have

$$
\left\langle\delta g^{2}\right\rangle=\frac{2}{15}
$$

It is interesting to recover the last two results on the average conductivity (or conductance) and its fluctuation using a systematic expansion of the recurrence time. To that purpose, we define the spectral determinant $S(s)$ by $S(s)=\operatorname{det}(-\Delta+s)$ where $\Delta$ is the Laplacian operator. Then, by definition of $Z(t)$, we have the relation

$$
T_{R}(s)=\int_{0}^{\infty} Z(t) e^{-s t} d t=\frac{\partial}{\partial s} \ln S(s)
$$

up to a regularization independent of the Laplace variable $s$. This relation is valid for all space dimensionality. Consider now as a working example [5 the case of a $1 d$ diffusive wire of length $L$. In order to describe the case of a perfect coupling of the wire to the reservoir, we demand Dirichlet boundary conditions for the diffusion equation. This is in contrast to the Schrödinger equation for which this choice corresponds to Neumann boundary conditions. Then, the spectral determinant $S(s)$ of the diffusion equation can be readily calculated and it is given by

$$
S(x)=\frac{\sqrt{x}}{\sinh \sqrt{x}}
$$

with $x=s \tau_{D}$. The average conductance $\delta g$ deduced from (68) and the fluctuation $\left\langle\delta g^{2}\right\rangle$ can be written generally as

$$
\begin{aligned}
\delta g & =2 \frac{\partial}{\partial x} \ln S(x)=-\frac{1}{3} \\
\left\langle\delta g^{2}\right\rangle & =12 \frac{\partial^{2}}{\partial x^{2}} \ln S(x)=\frac{2}{15}
\end{aligned}
$$

In the limit $x \rightarrow 0$, we can expand the spectral determinant namely $\ln S(x) \simeq_{x \rightarrow 0}-\frac{x}{6}+\frac{x^{2}}{180}$ and recover the previous result.

\section{Example 2. Multiple scattering of light: the albedo}

We shall now give an example of the use of the quantum probability taken from the multiple scattering of electromagnetic waves in disordered suspensions. To that purpose, consider the scattering medium as being the half-space $z \geq 0$. The other half-space is a free medium which contains both the sources of the waves and the detectors. We also assume that both incident and emergent waves are plane waves with respective wavevectors $\mathbf{k}_{\mathbf{i}}=k \hat{\mathbf{s}}_{\mathbf{i}}$ and $\mathbf{k}_{\mathbf{e}}=k \hat{\mathbf{s}}_{\mathbf{e}}$, where $\hat{\mathbf{s}}_{\mathbf{i}}$ and $\hat{\mathbf{s}}_{\mathbf{e}}$ are unit vectors. The waves experience only elastic scattering in the medium so that after a collision, only the direction $\hat{\mathbf{s}}$ changes while the amplitude $k=\frac{\omega_{0}}{c}$ remains constant. The reflection coefficient $\alpha\left(\hat{\mathbf{s}}_{\mathbf{i}}, \hat{\mathbf{s}}_{\mathbf{e}}\right)$ for this geometry is called the albedo and is proportional to the intensity $I\left(\mathbf{R}, \hat{\mathbf{s}}_{\mathbf{i}}, \hat{\mathbf{s}}_{\mathbf{e}}\right)$ emerging from the medium per unit surface and per unit solid angle measured at a point $\mathbf{R}$ at infinity. If $F_{\text {inc }}$ defines the flux of the incident beam (related to the incident intensity $I_{\text {inc }}$ by $F_{\text {inc }}=c S I_{\text {inc }}$ ) where $S$ is the illuminated surface in the plane $z=0$, then the albedo is given by

$$
\alpha\left(\hat{\mathbf{s}}_{\mathbf{i}}, \hat{\mathbf{s}}_{\mathbf{e}}\right)=\frac{R^{2} c}{F_{\text {inc }}} I\left(\mathbf{R}, \hat{\mathbf{s}}_{\mathbf{i}}, \hat{\mathbf{s}}_{\mathbf{e}}\right)
$$

In order to calculate the intensity $I\left(\mathbf{R}, \hat{\mathbf{s}}_{\mathbf{i}}, \hat{\mathbf{s}}_{\mathbf{e}}\right)$, we need to calculate first the radiative solutions of the Helmholtz equation at the Fraunhoffer approximation. It is given by

$$
\psi_{\omega_{0}}\left(\hat{\mathbf{s}}_{\mathbf{i}}, \hat{\mathbf{s}}_{\mathbf{e}}\right)=\int d \mathbf{r} d \mathbf{r}^{\prime} e^{i k\left(\hat{\mathbf{s}}_{\mathbf{i}} \cdot \mathbf{r}-\hat{\mathbf{s}}_{\mathbf{e}} \cdot \mathbf{r}^{\prime}\right)} G\left(\mathbf{r}, \mathbf{r}^{\prime}, \omega_{0}\right)
$$


and the intensity is then $I\left(\mathbf{R}, \hat{\mathbf{s}}_{\mathbf{i}}, \hat{\mathbf{s}}_{\mathbf{e}}\right)=\left|\psi_{\omega_{0}}\left(\hat{\mathbf{s}}_{\mathbf{i}}, \hat{\mathbf{s}}_{\mathbf{e}}\right)\right|^{2}$. Before averaging over the disorder, the albedo looks like a random pattern of bright and dark spots. This is a speckle pattern [12, 13] whose long range correlations are a specific feature of multiple scattering. By averaging over the disorder (either liquid suspension [14, 15, 16] or rotating solid sample [17]), the speckle is washed out and the two surviving contributions are respectively associated to the diffuson and the cooperon.

\subsection{The incoherent albedo: the diffuson}

We have calculated previously the various contributions to the intensity. The incoherent part is given by the diffuson contribution, namely the relation (49), where we take as the source term the incident plane wave

$$
\bar{\psi}_{i}\left(\mathbf{r}_{1}\right)=\sqrt{\frac{c I_{i n c}}{4 \pi}} e^{-\left|\mathbf{r}_{1}-\mathbf{r}\right| / 2 l_{e}} e^{-i k \hat{\mathbf{s}}_{\mathbf{i}} \cdot \mathbf{r}_{1}}
$$

where $\mathbf{r}$ is the impact point on the surface $z=0$ and $\mathbf{r}_{1}$ is the position of the first collision. The albedo rewrites

$$
\alpha_{d}=\frac{R^{2}}{S} \int d \mathbf{r}_{1} d \mathbf{r}_{2}\left|\bar{G}^{R}\left(\mathbf{r}_{2}, \mathbf{R}\right)\right|^{2} \Gamma\left(\mathbf{r}_{1}, \mathbf{r}_{2}\right) e^{-\left|\mathbf{r}_{1}-\mathbf{r}\right| / l_{e}}
$$

The Fraunhoffer approximation consists in taking the limit $\left|\mathbf{R}-\mathbf{r}_{2}\right| \rightarrow \infty$, so that the Green function $\bar{G}^{R}\left(\mathbf{r}_{2}, \mathbf{R}\right)$ can be expanded as

$$
\begin{aligned}
\bar{G}^{R}\left(\mathbf{r}_{2}, \mathbf{R}\right) & =e^{-\left|\mathbf{r}^{\prime}-\mathbf{r}_{2}\right| / 2 l_{e}} \frac{e^{i k\left|\mathbf{R}-\mathbf{r}_{2}\right|}}{4 \pi\left|\mathbf{R}-\mathbf{r}_{2}\right|} \\
& \simeq e^{-\left|\mathbf{r}^{\prime}-\mathbf{r}_{2}\right| / 2 l_{e}} e^{-i k \hat{\mathbf{s}}_{\mathbf{e}} \cdot \mathbf{r}_{2}} \frac{e^{-i k R}}{4 \pi R}
\end{aligned}
$$

By defining the respective projections $\mu$ and $\mu_{0}$ of the vectors $\hat{\mathbf{s}}_{\mathbf{i}}$ and $\hat{\mathbf{s}}_{\mathbf{e}}$ on the $z$ axis, we obtain finally

$$
\alpha_{d}=\frac{1}{(4 \pi)^{2} S} \int d \mathbf{r}_{1} d \mathbf{r}_{2} e^{-\frac{z_{1}}{\mu_{0} l_{e}}} e^{-\frac{z_{2}}{\mu l_{e}}} \Gamma\left(\mathbf{r}_{1}, \mathbf{r}_{2}\right)
$$

This expression calls for a number of remarks. We first notice that it does not contain any dependence on the direction namely on the incident and emerging vectors $\hat{\mathbf{s}}_{\mathbf{i}}$ and $\hat{\mathbf{s}}_{\mathbf{e}}$. Therefore, at this approximation, the albedo is flat i.e. does not have any angular structure. Second, if we compare this expression to its counterpart for the conductivity, we see that although both of them describe transport, the conductivity vanishes for the diffuson while it does not for the albedo.

The structure factor $\Gamma$ is related to the diffusion probability through the relation

$$
P_{d}\left(\mathbf{r}_{1}, \mathbf{r}_{2}\right)=\frac{l_{e}^{2}}{4 \pi c} \Gamma\left(\mathbf{r}_{1}, \mathbf{r}_{2}\right)
$$

so that we obtain

$$
\alpha_{d}=\frac{c}{4 \pi l_{e}^{2}} \int_{0}^{\infty} d z_{1} d z_{2} e^{-\frac{z_{1}}{\mu_{0} l_{e}}} e^{-\frac{z_{2}}{\mu l_{e}}} \int_{S} d^{2} \rho P_{d}\left(z_{1}, z_{2}, \rho\right)
$$

where now due to the geometry of the medium, the function $P_{d}$ depends on $z_{1}, z_{2}$ and the projection $\rho$ of the vector $\mathbf{r}_{1}-\mathbf{r}_{2}$ onto the plane $z=0$. Within the diffusion approximation, $P_{d}$ can be calculated for this geometry using the image method [18, 19]. This gives

$$
P_{d}\left(\mathbf{r}, \mathbf{r}^{\prime}\right)=\frac{1}{4 \pi D}\left[\frac{1}{\left|\mathbf{r}-\mathbf{r}^{\prime}\right|}-\frac{1}{\left|\mathbf{r}-\mathbf{r}^{\prime *}\right|}\right]
$$

where $\mathbf{r}^{\prime *}=\left(r^{\prime *},-z^{\prime}+2 z_{0}\right)$ and $z_{0}=\frac{2}{3} l_{e}$. Then, it appears that $P_{d}$ does not vanish on the plane $z=0$ but instead for $z=-z_{0}$. It is possible for that geometry to compare the validity of the diffusion approximation with the known exact solution for the semi-infinite problem without sources (the so called Milne problem) that can be solved using the Wiener-Hopf method [20]. There, we obtain instead that $P_{d}$ vanishes on the plane $z=-0.7104 l_{e}$. This justifies the use of 
the diffusion approximation. But, it is important at this point to make two important remarks. First, it would be a mistake to believe that on the basis of the two examples, infinite and semiinfinite spaces, for which the diffusion approximation works well that it is indeed always the case. The generalization and the validity of the diffusion equation in a restricted geometry is a difficult problem. Second, all the expressions we have obtained so far are valid for isotropic scatterers for which there is no difference between the elastic and the transport mean free paths. When relaxing this approximation, namely dealing with anisotropic scattering, we have to include both of them. Although it appears clearly from simple physical considerations that in the expression (82), we must leave the elastic mean free path $l_{e}$ in the exponential factors that describe the first and last collisions, and replace it by the transport mean free path in the expression of $P_{d}$, this point needs further investigation using for instance transport theory [6, 19].

Finally, by replacing (83) into (82), we obtain

$$
\alpha_{d}=\frac{3}{4 \pi} \mu \mu_{0}\left(\frac{z_{0}}{l_{e}}+\frac{\mu \mu_{0}}{\mu+\mu_{0}}\right)
$$

\subsection{The coherent albedo: the cooperon}

Along the same lines, we can now evaluate the contribution of the cooperon to the albedo. Using the expression (50) for the intensity, we obtain

$$
\alpha_{c}\left(\hat{\mathbf{s}}_{\mathbf{i}}, \hat{\mathbf{s}}_{\mathbf{e}}\right)=\frac{1}{(4 \pi)^{2} S} \int d \mathbf{r}_{1} d \mathbf{r}_{2} e^{-\frac{1}{2}\left(\frac{1}{\mu}+\frac{1}{\mu_{0}}\right) \frac{z_{1}+z_{2}}{l_{e}}} \Gamma\left(\mathbf{r}_{1}, \mathbf{r}_{2}\right) e^{i k\left(\hat{\mathbf{s}}_{\mathbf{i}}+\hat{\mathbf{s}}_{\mathbf{e}}\right) \cdot\left(\mathbf{r}_{2}-\mathbf{r}_{1}\right)}
$$

Unlike the previous case, there is now a phase term present in this expression which is at the origin of a new angular structure of the albedo. It is straightforward to check that, assuming $\hat{\mathbf{s}}_{\mathbf{i}}+\hat{\mathbf{s}}_{\mathbf{e}}=\mathbf{0}$, i.e. measuring the albedo right in the backscattering direction, we have

$$
\alpha_{c}(\theta=0)=\alpha_{d}
$$

where the angle $\theta$ is between the directions $\hat{\mathbf{s}}_{\mathbf{i}}$ and $\hat{\mathbf{s}}_{\mathbf{e}}$. Then, the total albedo $\alpha(\theta)=\alpha_{d}+\alpha_{c}(\theta)$ is such that

$$
\alpha(\theta=0)=2 \alpha_{d}
$$

Using the expression (83) for the probability and integrating, we obtain

$$
\alpha_{c}(\theta)=\frac{3}{8 \pi} \frac{\mu \mu_{0}}{\left(1+\mu k_{\perp} l_{e}\right)\left(1+\mu_{0} k_{\perp} l_{e}\right)}\left(\frac{1-e^{-2 k_{\perp} z_{0}}}{k_{\perp} l_{e}}+2 \frac{\mu \mu_{0}}{\mu+\mu_{0}}\right)
$$

in this expression, $\mathbf{k}_{\perp}=\left(\mathbf{k}_{\mathbf{i}}+\mathbf{k}_{\mathbf{e}}\right)_{\perp}=k\left(\hat{\mathbf{s}}_{\mathbf{i}}+\hat{\mathbf{s}}_{\mathbf{e}}\right)_{\perp}$ is the projection onto the plane $x O y$ of the vector $\mathbf{k}_{\mathbf{i}}+\mathbf{k}_{\mathbf{e}}$. At small angles, we have $k_{\perp} \simeq \frac{2 \pi}{\lambda} \theta$, while at large angles, $\alpha_{c}(\theta \rightarrow \infty)=0$. Then, the coherent contribution to the albedo is finite only in a cone of angular aperture $\frac{\lambda}{2 \pi l_{e}}$ around the backscattering direction. By expanding near $\theta=0$ we obtain the expression

$$
\alpha_{c}(\theta) \simeq \alpha_{d}-\frac{3}{4 \pi} \frac{\left(l_{e}+z_{0}\right)^{2}}{l_{e}} k_{\perp}+O\left(k_{\perp}\right)^{2}
$$

Thus, the albedo shows a cusp near $\theta=0$ namely, its derivative is discontinuous at this point.

\section{Example 3: Spectral correlations}

In the previous two examples, we discussed transport coefficients. They give a description of the system when it is connected to another "reference" medium. There is another characterization independent of the coupling to another system, which focuses on spectral properties i.e. properties of the energy spectrum of the solutions of the wave equations. For electronic systems, they are related to the equilibrium thermodynamic properties like the magnetization of a metallic sample. 
For disordered systems, it also raises an important issue: these systems are part of the larger class of complex systems, usually non integrable. The complete set of correlation functions of the energy levels provides a complete description of the thermodynamic properties. For instance, it is possible just by inspection of the energy spectrum to determine whether or not an electronic system is a good metal or an Anderson insulator.

Let us start by defining the set of eigenenergies $\epsilon_{\alpha}$ of the Schrödinger (or Helmholtz) equation in a confined geometry (e.g. a box). The density of states per unit volume is given by

$$
\rho(\epsilon)=\frac{1}{\Omega} \sum_{\alpha} \delta\left(\epsilon-\epsilon_{\alpha}\right)
$$

It can be written as well in terms of the Green function for the Schrödinger equation, using the equality

$$
\rho(\epsilon)=-\frac{1}{\pi \Omega} \operatorname{Im} \int d \mathbf{r} G^{R}(\mathbf{r}, \mathbf{r}, \epsilon)
$$

By averaging over the disorder, we define the various correlation functions. For instance, the two-point correlation function is

$$
\overline{\rho\left(\epsilon_{1}\right) \rho\left(\epsilon_{2}\right)}-\bar{\rho}\left(\epsilon_{1}\right) \bar{\rho}\left(\epsilon_{2}\right)
$$

where the average density of states coincides with its free value $\rho_{0}$ and therefore is related to the mean level spacing $\Delta$ through $\bar{\rho}=\frac{1}{\Delta \Omega}$. It is important at this point to make the following remark. The average we consider here is over the random potential (伍). We could have taken as well the average over different parts of the spectrum as is done for instance in the quantum description of classically chaotic billiards where there is no random potential to average over. In the diffusion approximation, it has been shown numerically that these two ways to average are equivalent [4, 21] but this not need to be the general case. We then define the dimensionless correlation function

$$
K(\omega)=\frac{\overline{\rho(\epsilon) \rho(\epsilon-\omega)}}{\bar{\rho}(\epsilon)^{2}}-1
$$

It can be expressed in terms of the Green function under the form

$$
K(\omega)=\Delta^{2} \int d \mathbf{r} d \mathbf{r}^{\prime} K\left(\mathbf{r}, \mathbf{r}^{\prime}, \omega\right)
$$

with

$$
K\left(\mathbf{r}, \mathbf{r}^{\prime}, \omega\right)=\frac{1}{2 \pi^{2}} \operatorname{Re}{\overline{G^{R}(\mathbf{r}, \mathbf{r}, \epsilon) G^{A}\left(\mathbf{r}^{\prime}, \mathbf{r}^{\prime}, \epsilon-\omega\right)}}^{c}
$$

where $\bar{\ldots}^{c}$ represents the cumulant average. The diffuson and cooperon contributions to the local function $K\left(\mathbf{r}, \mathbf{r}^{\prime}, \omega\right)$ can be expressed in terms of $P_{d}$ and the structure factor as 22]

$$
K_{d}\left(\mathbf{r}, \mathbf{r}^{\prime}, \omega\right)=\frac{1}{2 \pi^{2}} \operatorname{Re}\left[P_{d}\left(\mathbf{r}, \mathbf{r}^{\prime} \omega\right) P_{d}\left(\mathbf{r}^{\prime}, \mathbf{r}, \omega\right)\right]
$$

while

$$
K_{c}\left(\mathbf{r}, \mathbf{r}^{\prime}, \omega\right)=2 \rho_{0}^{2} \tau_{e}^{4} \operatorname{Re}\left[\Gamma_{\omega}^{\prime}\left(\mathbf{r}, \mathbf{r}^{\prime}\right) \Gamma_{\omega}^{\prime}\left(\mathbf{r}^{\prime}, \mathbf{r}\right)\right]
$$

when the system has time-reversal invariance. Finally, we define the Fourier transform $\tilde{K}(t)$ which is often called the form factor. By collecting the two previous contributions, it can be written

$$
\tilde{K}(t)=\frac{\Delta^{2}}{4 \pi^{2}}|t| Z(|t|)=\frac{\Delta^{2}}{4 \pi^{2}}|t| \int_{\Omega} P_{d}(\mathbf{r}, \mathbf{r},|t|) d \mathbf{r}
$$

which is precisely the form that is obtained assuming the Random Matrix Theory [4, 21] provided we consider the regime for which $\hbar / \Delta \gg t \gg \tau_{D}$ where $\tau_{D}=L^{2} / D$. The first inequality which involves the Heisenberg time $\hbar / \Delta$ enforces the condition of a continuous spectrum while the second one indicates that we must be in the ergodic limit where the diffusing particle explored the whole system. 


\section{Conclusion}

We have presented in this short review a very partial selection of highlights in the field of multiple scattering of waves in disordered media. Our aim was more to give a feeling of the profound unity of the physical phenomena and therefore of the methods to handle them rather than discussing an extensive list of effects. Among them, we should mention the study of fluctuations of the intensity for waves both in the weak disorder regime and near the Anderson transition that will be covered in details by the reviews of R. Pnini, A. Genack and G.Maret. This is certainly a problem for which the recent developments have been very spectacular. We have studied the coherent albedo in the weak disorder case. It should be extended to the strong disorder limit. Finally, our last example on spectral correlations should be considered as another way to obtain the semiclassical results reviewed in the contribution of D. Delande.

\section{References}

[1] These notes are a partial and preliminary account of the monography Cohérence et diffusion dans les milieux désordonnés by E. Akkermans and G. Montambaux, to be published by CNRS Intereditions for the french version.

[2] S. Chandrasekhar, Radiative transfer (Dover, N.Y. 1960).

[3] S. Chakraverty and A. Schmid, Phys. Rep. 140,193 (1986)

[4] G. Montambaux, in Quantum fluctuations, proceedings of the Les Houches Summer School, Session LXIII, ed. by S. Reynaud et al. (North Holland, Amsterdam, 1996), p.387.

[5] M. Pascaud and G. Montambaux, Phys. Rev. Lett. 82 (1999), 4512 and Phys. Uspekhi 41,182 (1998).

[6] A. Ishimaru, Wave propagation and scattering in random media, Vol.I,II (Academic, N.Y. 1978).

[7] S. Doniach et E.H. Sondheimer, Green's Functions for Solid State Physicists, Frontiers in Physics, W.A. Benjamin, (1974)

[8] G. Bergmann, Phys. Rep. 107, 1 (1984)

[9] J. Rammer, Quantum transport theory, Frontiers in Physics, Perseus books (1998)

[10] Mesoscopic quantum physics, proceedings of the Les Houches Summer School, Session LXI, ed. by E.Akkermans, G. Montambaux, J.L. Pichard and J. Zinn-Justin (North Holland, Amsterdam, 1995).

[11] C. Itzykson and J.M. Drouffe, Statistical field theory, Vol. 1 (Cambridge, 1989)

[12] C. Dainty ed., Laser speckle and related phenomena, Topics in a applied physics, Vol.9 (Springer, Berlin, 1984).

[13] G. Maret, in Mesoscopic quantum physics, proceedings of the Les Houches Summer School, Session LXI, ed. by E.Akkermans, G. Montambaux, J.L. Pichard and J. ZinnJustin (North Holland, Amsterdam, 1995).

[14] Y. Kuga and A. Ishimaru, J.Opt.Soc. Am., A8, 831 (1984)

[15] M.P. van Albada et A. Lagendijk, Phys. Rev. Lett. 55 (1985), 2692.

[16] P.E. Wolf et G. Maret, Phys. Rev. Lett. 55 (1985), 2696. 
[17] M. Kaveh, M. Rosenbluh, I. Edrei et I. Freund, Phys. Rev. Lett. 57 (1986), 2049

[18] E. Akkermans, P.E. Wolf, and R. Maynard, Phys. Rev. Lett. 56 (1986), 1471-1474

[19] E. Akkermans, P.E. Wolf, R. Maynard and G. Maret, J. de Physique (France), 49 (1988), $77-98$

[20] H. C. van de Hulst, Multiple light scattering (Dover, N.Y. 1981).

[21] O. Bohigas, in Chaos and Quantum Physics, Proceedings of the Les Houches Summer School, Session LII, ed. by M.J. Giannoni, A. Voros and J. Zinn-Justin (North Holland, Amsterdam, 1991), p.91

[22] B.L. Al'tshuler and B. Shklovskiı̌, Sov. Phys. JETP 64, 127 (1986). 